PROCEEDINGS OF THE

AMERICAN MATHEMATICAL SOCIETY

Volume 136, Number 7, July 2008, Pages 2313-2321

S 0002-9939(08)09198-3

Article electronically published on February 28, 2008

\title{
A NOTE ON THE INJECTIVE DIMENSION OF LOCAL COHOMOLOGY MODULES
}

\author{
M. HELLUS
}

(Communicated by Bernd Ulrich)

\begin{abstract}
For a Noetherian ring $R$ we call an $R$-module $M$ cofinite if there exists an ideal $I$ of $R$ such that $M$ is $I$-cofinite; we show that every cofinite module $M$ satisfies $\operatorname{dim}_{R}(M) \leq \operatorname{injdim}_{R}(M)$. As an application we study the question which local cohomology modules $H_{I}^{i}(R)$ satisfy $\operatorname{injdim}_{R}\left(H_{I}^{i}(R)\right)=$ $\operatorname{dim}_{R}\left(H_{I}^{i}(R)\right)$. There are two situations where the answer is positive. On the other hand, we present two counterexamples, the failure in these two examples coming from different reasons.
\end{abstract}

\section{INTRODUCTION}

Let $I$ be an ideal of a Noetherian ring $R$. By definition, an $R$-module $M$ is $I$ cofinite if $\operatorname{Supp}_{R}(M) \subseteq \mathcal{V}(I)$ and if all $\operatorname{Ext}_{R}^{i}(R / I, M)$ are finitely generated. Note that, for local $(R, m), m$-cofiniteness is the same as being Artinian. The concept of $I$-cofiniteness was introduced by Hartshorne (4]); see [1, 5, 7, 11, 12, for more material on cofiniteness.

Let $M$ be a module over a ring $R$. An injective resolution of $M$ is an exact sequence

$$
0 \rightarrow M \rightarrow E^{0} \rightarrow E^{1} \rightarrow \ldots \quad,
$$

where all $E^{i}$ are injective $R$-modules. By definition, $\operatorname{injdim}_{R}(M) \leq n$ if there is an injective resolution of $M$ such that $0=E^{n+1}=E^{n+2}=\ldots$.

Every finitely generated $M$ over a Noetherian ring $R$ satisfies $\operatorname{dim}_{R}(M) \stackrel{(1)}{\leq}$ $\operatorname{injdim}_{R}(M)$; if $R$ is local and $\operatorname{injdim}_{R}(M)<\infty$ holds, one has $\operatorname{injdim}_{R}(M) \stackrel{(2)}{=}$ $\operatorname{depth}(R)$. Both (1) and (2) are known as the Bass formula. In this work (Theorem 2.3) we generalize (1) to the class of all $R$-modules that are $I$-cofinite for at least one ideal $I$ of $R$ (we call these modules cofinite modules), where $R$ is a Noetherian ring. We show (Remark 2.2) that (2) does not generalize to the class of all cofinite modules and explain why this generalization fails.

In the sequel we prove some consequences of Theorem 2.3 for local cohomology modules; in particular we are dealing with the following:

Received by the editors October 26, 2006, and, in revised form, February 28, 2007, and April $2,2007$.

2000 Mathematics Subject Classification. Primary 13D45; Secondary $13 \mathrm{C} 05$.

Key words and phrases. Local cohomology, injective dimension, Bass formula.

(C)2008 American Mathematical Society Reverts to public domain 28 years from publication 
Question. When does

$$
\operatorname{injdim}_{R}\left(H_{I}^{i}(R)\right)=\operatorname{dim}_{R}\left(H_{I}^{i}(R)\right)
$$

hold?

(Here $I$ is an ideal of a Noetherian ring $R$ and $H_{I}^{i}(R)$ is the $i$-th local cohomology of $R$ supported in $I$.) There is a positive answer in the following two situations (see Corollary 2.6 and Remark 2.7):

- $R$ is a Noetherian local regular ring containing a field and $H_{I}^{i}(R)$ is $I$ cofinite.

- $R$ is a Noetherian local Gorenstein $\operatorname{ring} R$ and $H_{I}^{n}(R)=0$ for every $n \neq i$.

Finally we prove that the answer to the above question is negative in general. More precisely, we present two counterexamples which are different in nature (Example 2.9 and the last example from 2.11). In 2.12 we summarize answers to the above question.

By $E_{R}(M)$ we denote a fixed $R$-injective hull of an $R$-module $M$.

\section{Results}

2.1. A positive result. The following statement is contained in [1, Corollary 1]. For the sake of completeness we present a simple proof:

Lemma 2.1. Let $R$ be a Noetherian ring, $I \subseteq R$ an ideal and $M$ an $I$-cofinite $R$-module. Then, for every ideal $J$ of $R$ containing $I$ and for every $l \in \mathbb{N}$, the module $\operatorname{Ext}_{R}^{l}(R / J, M)$ is finitely generated.

Proof. By induction on $l$ : The case $l=0$ is trivial, because for every ideal $J$ of $R$ containing $I$ there is an inclusion $\operatorname{Hom}_{R}(R / J, M) \subseteq \operatorname{Hom}_{R}(R / I, M)$ and the latter module is finitely generated. We assume $l>0$ and that the statement is true for smaller $l$ : We choose $x_{1}, \ldots, x_{n} \in R$ such that $J=I+\left(x_{1}, \ldots, x_{n}\right) R$ and prove our induction hypothesis by induction on $n$. In the case $n=0$ there is nothing to prove. We assume $n>0$ and that the statement holds for smaller $n$. Consider the short exact sequence

$$
0 \rightarrow R /\left(I_{n-1}:_{R} x_{n}\right) \stackrel{x_{n}}{\rightarrow} R / I_{n-1} \rightarrow R / J \rightarrow 0
$$

(we define $I_{s}=I+\left(x_{1}, \ldots, x_{s}\right) R$ for every $s \in\{1, \ldots, n\}$ ), where $x_{n}$ denotes multiplication by $x_{n}$ on $R / I_{n-1}$. We get an exact sequence

$$
\operatorname{Ext}_{R}^{l-1}\left(R /\left(I_{n-1}:_{R} x_{n}\right), M\right) \rightarrow \operatorname{Ext}_{R}^{l}(R / J, M) \rightarrow \operatorname{Ext}_{R}^{l}\left(R / I_{n-1}, M\right) .
$$

The latter module in this sequence is finitely generated by induction on $n$, and the first module is finitely generated by induction on $l$. Therefore, the second module is finitely generated; both inductions are completed.

Remark 2.2. If $(R, m)$ is a Noetherian local ring and $M$ is a finitely generated $R$-module of finite injective dimension, then $\operatorname{injdim}_{R}(M) \stackrel{(2)}{=} \operatorname{depth}(R)$. This is not true for every cofinite $R$-module $M$; here is a counterexample. Let $R=k[[X]]$ be a formal power series ring in one variable over a field $k$; set $M=k\left[X^{-1}\right]=$ $k \cdot 1 \oplus k \cdot X^{-1} \oplus \ldots$ It is well known that $M$, together with its natural $R$-module structure, is an $R$-injective hull of $k$. Clearly, $M$ is Artinian, i.e. $X R$-cofinite, and we have

$$
\operatorname{injdim}_{R}(M)=0 \neq 1=\operatorname{depth}(R) .
$$


More generally, Remark 2.7 below will present a class of local cohomology modules $M$ for which

$$
\operatorname{injdim}_{R}(M)=\operatorname{dim}_{R}(M)
$$

holds, and for which $\operatorname{dim}_{R}(M)$ is not equal to $\operatorname{depth}(R)$ in general.

If $R$ is a Noetherian ring, every finitely generated $R$-module $M$ satisfies

$$
\operatorname{dim}_{R}(M) \leq \operatorname{injdim}_{R}(M) .
$$

The same formula holds for any cofinite $R$-module $M$ (by definition, $M$ is cofinite if there exists an ideal $I$ of $R$ such that $M$ is $I$-cofinite).

Theorem 2.3. Let $R$ be a Noetherian ring and $M$ a cofinite $R$-module. Then

$$
\operatorname{dim}_{R}(M) \leq \operatorname{injdim}_{R}(M)
$$

holds.

Proof. Let $I$ be an ideal of $R$ such that $M$ is $I$-cofinite. It suffices to show the following two statements (by $\mu_{k}(p, M):=\operatorname{rank}_{R_{p} / p R_{p}}\left(\operatorname{Ext}_{R_{p}}^{k}\left(R_{p} / p R_{p}, M_{p}\right)\right.$ ) we denote the $k$-th Bass number of $M$ with respect to a prime ideal $p$ of $R$ ).

(a) If $p$ is minimal in $\operatorname{Supp}_{R}(M)$, then $\mu_{0}(p, M) \neq 0$.

(b) If $p \subsetneq p^{\prime}$ are prime ideals of $R$ with no prime in between such that $\mu_{k}(p, M) \neq$ 0 , then $\mu_{k+1}\left(p^{\prime}, M\right) \neq 0$.

Let $0 \rightarrow M \rightarrow E^{\bullet}$ be a minimal injective resolution of $M$. Statement (a) is clear, because for every $p$ minimal in $\operatorname{Supp}_{R}(M)$ one has $p \in \operatorname{Ass}_{R}(M)=\operatorname{Ass}_{R}\left(E^{0}\right)$, i.e. $\mu_{0}(p, M) \neq 0$. Proof of (b): Localizing at $p^{\prime}$ allows us to assume that $(R, m)$ is local and $p^{\prime}=m$. Choose any $x \in m \backslash p$. The short exact sequence

$$
0 \rightarrow R / p \stackrel{x}{\rightarrow} R / p \rightarrow R /(p+x R) \rightarrow 0
$$

induces an exact sequence

$$
\operatorname{Ext}_{R}^{k}(R / p, M) \stackrel{x}{\rightarrow} \operatorname{Ext}_{R}^{k}(R / p, M) \rightarrow \operatorname{Ext}_{R}^{k+1}(R /(p+x R), M) .
$$

Now, $\mu_{k}(p, M) \neq 0$ implies $p \in \operatorname{Supp}_{R}(M)$ (note that one has $\operatorname{Supp}_{R}\left(E^{l}\right) \subseteq$ $\operatorname{Supp}_{R}(M)$ for every $l$ as $E^{\bullet}$ is a minimal injective resolution of $\left.M\right)$ and, therefore, $p \supseteq I$. By Lemma 2.1 we conclude that $\operatorname{Ext}_{R}^{k}(R / p, M)(\neq 0)$ is a finitely generated $R$-module. This fact, together with our last exact sequence and the lemma of Nakayama implies that $\operatorname{Ext}_{R}^{k+1}(R /(p+x R), M) \neq 0$. The nonzero $R$-module $R /(p+x R)$ has finite length, and therefore it is easy to see that $\operatorname{Ext}_{R}^{k+1}(R / m, M) \neq$ 0, i.e. $\mu_{k+1}(m, M) \neq 0$.

Corollary 2.4. Let $R$ be a Noetherian ring, $I$ an ideal of $R$ and $M$ a finitely generated $R$-module. Then $H_{I}^{l}(M)$ is $I$-cofinite for every $l \in \mathbb{N}$ if one of the following two conditions holds:

$\left(c_{1}\right) \operatorname{dim}(R / I)=1$.

$\left(c_{2}\right)$ There exists $p \in \mathbb{N}$ such that $H_{I}^{i}(M)$ is finitely generated for every $i \neq p$ (see Remark 2.5 on this condition; also note that it would suffice to assume that $H_{I}^{i}(M)$ is $I$-cofinite for all $\left.i \neq p\right)$.

Consequently, by Theorem 2.3, one has

$$
\operatorname{dim}_{R}\left(H_{I}^{l}(M)\right) \leq \operatorname{injdim}_{R}\left(H_{I}^{l}(M)\right)
$$

for every natural $l$. 
Proof. This follows immediately from Theorem 2.3 and the fact that $H_{I}^{l}(M)$ is $I$-cofinite for every $l \in \mathbb{N}$ if $\left(c_{1}\right)$ or $\left(c_{2}\right)$ holds; this was shown in [1, Theorem 1], resp. [12, Theorem 1.1], in the case of $\left(c_{1}\right)$ and in [11, Proposition 2.5] in the case of $\left(c_{2}\right)$.

Remark 2.5. In the situation of Corollary 2.4 set

$$
h:=\operatorname{height}\left(\left(I+\operatorname{Ann}_{R}(M)\right) / \operatorname{Ann}_{R}(M)\right)
$$

and

$$
c:=\operatorname{cd}(I, M)=\operatorname{cd}\left(I, R / \operatorname{Ann}_{R}(M)\right) .
$$

Note that $\operatorname{cd}(I, M):=\sup \left\{\ell \in \mathbb{N} \mid H_{I}^{\ell}(M) \neq 0\right\}$ is the cohomological dimension of $I$ on $M$; also note that $0 \leq h \leq c$. One has

$$
c>0 \Rightarrow H_{I}^{c}(M) \text { is not finitely generated. }
$$

(Proof: By localizing in any prime ideal of the support of $H_{I}^{c}(M)$ we may assume that $(R, m)$ is local; the functor $H_{I}^{c}$ is right exact on modules whose support is contained in $V\left(A n n_{R}(M)\right)$ and therefore one has $H_{I}^{c}(M) \otimes_{R} R / m=H_{I}^{c}(M / m M)=$ 0 , as $c$ is positive; by Nakayama, $H_{I}^{c}(M)$ is not finite.) Also,

$$
h>0 \Rightarrow H_{I}^{h}(M) \text { is not finitely generated. }
$$

(Proof: We assume that $H_{I}^{h}(M)$ is finitely generated. Let $p$ be a prime ideal of $R$ containing $I+\operatorname{Ann}_{R}(M)$ such that height $\left(p / \operatorname{Ann}_{R}(M)\right)=h$. Then $I R_{p} /$ $\operatorname{Ann}_{R}(M) R_{p}$ is $p R_{p} / \operatorname{Ann}_{R}(M) R_{p}$-primary and we conclude that

$$
H_{I}^{h}(M)_{p}=H_{p R_{p}}^{h}\left(M_{p}\right)
$$

is finite as an $R_{p}$-module; by construction, $\operatorname{dim}_{R_{p}}\left(M_{p}\right)=h$ and our above result on $c$ shows that $H_{p R_{p}}^{h}\left(M_{p}\right)$ is not finite, a contradiction.) Thus, if $h>0$, the equality $p=h=c$ is necessary for condition $\left(c_{2}\right)$ of Corollary 2.4.

\subsection{An application and some examples.}

Corollary 2.6. Let $R$ be a Noetherian local regular ring containing a field and $I$ an ideal of $R$ such that $H_{I}^{l}(R)$ is $I$-cofinite for some natural number $l$. Then

$$
\operatorname{injdim}_{R}\left(H_{I}^{l}(R)\right)=\operatorname{dim}_{R}\left(H_{I}^{l}(R)\right)
$$

holds. In particular, one has $\operatorname{inj}_{\operatorname{dim}}\left(H_{I}^{l}(R)\right)=\operatorname{dim}_{R}\left(H_{I}^{l}(R)\right)$ if one of the following conditions is satisfied:

(i) $\operatorname{dim}(R / I) \leq 1$.

(ii) The residue field $R / m$ (where $m$ denotes the maximal ideal of $R$ ) is separably closed, $\operatorname{dim}(R / I)=2, I$ is equidimensional and $\operatorname{Spec}(R / I) \backslash\{m\}$ is connected.

(iii) $I$ is a set-theoretic complete intersection.

(iv) The characteristic of $R$ and $R / I$ is Cohen-Macaulay.

Proof. The first statement follows immediately from Theorem 2.3 together with the following theorem, which is due to Lyubeznik ([9, 10]):

Theorem. Let $R$ be a regular ring containing a field and $I$ an ideal of $R$. Then, for every $l \in \mathbb{N}$,

holds.

$$
\operatorname{injdim}_{R}\left(H_{I}^{l}(R)\right) \leq \operatorname{dim}_{R}\left(H_{I}^{l}(R)\right)
$$


In the second part it is true in all four cases that there is only one nonvanishing local cohomology module of $R$ with support in $I$ (which is then necessarily $I$-cofinite by [11, Prop. 2.5]); this is clear for the cases (i) and (iii). In the case of (ii), it follows from [6, Theorem 2.9] and in the case of (iv) from the identity

$$
H_{I}^{l}(R)=\operatorname{dirlim}_{e \in \mathbb{N}} \operatorname{Ext}_{R}^{l}\left(R / I^{\left[p^{e}\right]}, R\right)
$$

(where $I^{\left[p^{e}\right]}$ denotes the $p^{e}$-th Frobenius power of $I$ and $l$ is an arbitrary natural number) together with the flatness of the Frobenius map over regular local rings of positive characteristic.

Under suitable assumptions, the result of Corollary 2.6 holds for more general rings:

Remark 2.7. Let $I$ be an ideal of a Gorenstein $\operatorname{ring} R$ and $l \in \mathbb{N}$ such that

$$
H_{I}^{i}(R)=0 \quad(i \neq l)
$$

holds. Then the formula

$$
\operatorname{injdim}_{R}\left(H_{I}^{l}(R)\right)=\operatorname{dim}_{R}\left(H_{I}^{l}(R)\right)(=\operatorname{dim}(R / I))
$$

holds.

Proof. The proof follows e.g. from [5, Prop. 3.1].

Question 2.8. When does $\operatorname{inj}_{\operatorname{dim}}\left(H_{I}^{i}(R)\right)=\operatorname{dim} H_{I}^{i}(R)$ hold? In the situations of Corollary 2.6 and Remark 2.7 this question has a positive answer.

But Corollary 2.6 in general becomes false if one does not assume that $H_{I}^{l}(R)$ is $I$-cofinite, as the following example shows:

Example 2.9. Let $R=k[[x, y, z]]$ be a power series ring over a field $k$ and let $I$ be the ideal $(x y, x z) R$ of $R$. Because of $I=x R \cap(y, z) R$ we have the Mayer-Vietoris sequence

$$
0 \rightarrow H_{(y, z) R}^{2}(R) \rightarrow H:=H_{I}^{2}(R) \rightarrow E \rightarrow 0
$$

( $E$ is a fixed $R$-injective hull of $k$ ) and hence an exact sequence

$$
\begin{aligned}
& \operatorname{Hom}_{R}\left(R /(y, z) R, H_{(y, z) R}^{2}(R)\right) \rightarrow \operatorname{Hom}_{R}(R /(y, z) R, H) \\
\rightarrow & \operatorname{Hom}_{R}(R /(y, z) R, E) \rightarrow \operatorname{Ext}_{R}^{1}\left(R /(y, z) R, H_{(y, z) R}^{2}(R)\right) .
\end{aligned}
$$

The first and the last term of the previous sequence are both finitely generated, since $H_{(y, z) R}^{2}(R)$ is $(y, z) R$-cofinite (the last term is zero in fact). Therefore, $\operatorname{Hom}_{R}(R /(y, z) R, H)$ is finite if and only if $\operatorname{Hom}_{R}(R /(y, z) R, E)$ is finite, but $\operatorname{Hom}_{R}(R /(y, z) R, E)$ is isomorphic to $E_{k[[x]]}(k)$ (with an $R$-module structure induced by $R \rightarrow R /(y, z) R \cong k[[x]])$ and it is well known that the latter module is not finitely generated. We conclude that $\operatorname{Hom}_{R}(R /(y, z) R, H)$ is not finitely generated; in particular, $\operatorname{Hom}_{R}(R / I, H)$ is not finitely generated, and $H$ is not I-cofinite.

By using Cech cohomology, it is clear that $H$ is the cokernel of the natural map

$$
R_{x y} \oplus R_{x z} \rightarrow R_{x y z}
$$

Thus, by using Cech cohomology also for $H_{(y, z) R}^{2}(R)$,

$$
H=H_{(y, z) R}^{2}(R)_{x}=H_{(y, z) R_{(y, z) R}}^{2}\left(R_{(y, z) R}\right)=E_{R}(R /(y, z) R)
$$


(the second equality follows because $(y, z) R_{x}$ is a maximal ideal of the localized

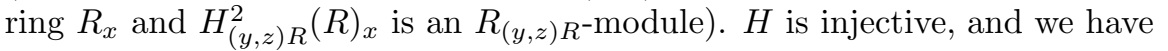

$$
\operatorname{injdim}_{R}(H)=0 \neq 1=\operatorname{dim}_{R}(H) .
$$

It seems that the negative answer to Question 2.8 in the previous example comes from the fact that the primary components of the ideal $I$ have different dimensions, but we will see that the answer is in general negative also if all components of $I$ have the same dimension. We start with some general facts:

Remark 2.10. There is a result from Huneke and Koh ([5, theorem 2.3(ii)]), resp. Lyubeznik (9, corollary 3.5]), which says that if $I$ is an ideal of a regular ring $R$ containing a field, then, for $l>\operatorname{bight}(I), \operatorname{Hom}_{R}\left(R / I, H_{I}^{l}(R)\right)$ is finitely generated only if $H_{I}^{l}(R)$ is zero. Thus, in order to find examples of non- $I$-cofinite local cohomology modules $H_{I}^{l}(R)$, one should consider ideals where $\operatorname{cd}(I, R)$ is big.

Faltings ([2]) proved upper bounds for the cohomological dimension $\operatorname{cd}(I, R)$ of an ideal $I$ of a regular local ring $R$ containing a field. Lyubeznik presents examples where these upper bounds are actually obtained ( $[$, and [ $[$, Corollary 5.3$])$ :

Theorem. Let $(R, m)$ be an excellent regular d-dimensional local ring containing a field. Let $s$ and $b$ be positive natural numbers such that $s=\lceil(d-1) / b\rceil$, where $\lceil\alpha\rceil$ is the maximum integer that does not exceed the real number $\alpha$. Let $I_{0}, \ldots, I_{s}$ be ideals of bigheight $b$ such that $I_{0}+\cdots+I_{s}$ is m-primary. Set $I=\bigcap_{0 \leq j \leq s} I_{j}$. Then $\operatorname{cd}(I, R)=d-s$ and this is precisely the maximal possible value (i.e., the general upper bound on $\operatorname{cd}(I, R)$ from [2]).

We investigate, with respect to Question 2.8, nontrivial examples where $s$ and $b$ are small (note that cases where the local cohomology module is cofinite are clear by Corollary 2.6); our last example will show that not all local cohomology modules $H$ arising from Lyubeznik's result above satisfy $\operatorname{injdim}_{R}(H)=\operatorname{dim}_{R}(H)$ :

Examples 2.11. Let $k$ be a field and $R=k\left[\left[x_{1}, \ldots, x_{d}\right]\right]$ a formal power series algebra over $k$ in $d$ variables.

- $s=2, b=2, d=5$. We take $I:=\left(x_{1}, x_{2}\right) R \cap\left(x_{3}, x_{4}\right) R \cap\left(x_{5}, x_{1}\right) R$ and $H:=H_{I}^{3}(R)$. Because of Remark 2.10 $H$ is not $I$-cofinite (it is not zero because, by Lyubeznik's result mentioned above, $\operatorname{cd}(R, I)=5-s=3)$. We claim that $H$ has a minimal injective resolution of the form

$$
\begin{aligned}
0 \rightarrow H \rightarrow E_{R}\left(R /\left(x_{1}, x_{2}, x_{3}, x_{4}\right) R\right) & \oplus E_{R}\left(R /\left(x_{1}, x_{3}, x_{4}, x_{5}\right) R\right) \\
\rightarrow E_{R}(R / m) & \rightarrow 0 .
\end{aligned}
$$

A part of a Mayer-Vietoris sequence with respect to the ideal $\left(x_{1}, x_{2}\right) R \cap$ $\left(x_{5}, x_{1}\right) R$ and $\left(x_{3}, x_{4}\right) R$ is:

$$
\begin{gathered}
H_{\left(x_{1}, x_{2}\right) R \cap\left(x_{5}, x_{1}\right) R}^{3}(R) \rightarrow H \\
\rightarrow H_{\left(x_{1}, x_{2}, x_{3}, x_{4}\right) R \cap\left(x_{1}, x_{3}, x_{4}, x_{5}\right) R}^{4}(R) \rightarrow H_{\left(x_{1}, x_{2}\right) R \cap\left(x_{5}, x_{1}\right) R}^{4}(R) .
\end{gathered}
$$

By another Mayer-Vietoris sequence argument, we see that in this sequence the first and last term vanish, and we get

$$
H=H_{\left(x_{1}, x_{2}, x_{3}, x_{4}\right) R \cap\left(x_{1}, x_{3}, x_{4}, x_{5}\right) R}^{4}(R) .
$$


For later use we note $\left\{\left(x_{1}, x_{2}, x_{3}, x_{4}\right) R,\left(x_{1}, x_{3}, x_{4}, x_{5}\right) R\right\} \stackrel{(+)}{\subseteq} \operatorname{Ass}_{R}(H)$. The Cech complex of $H$ with respect to $x_{2}, x_{5}$ has the form

$$
0 \rightarrow H \rightarrow H_{x_{2}} \oplus H_{x_{5}} \rightarrow H_{x_{2} \cdot x_{5}}=0 .
$$

The spectral sequence belonging to the composed functors

$$
\Gamma_{\left(x_{2}, x_{5}\right)} \circ \Gamma_{\left(x_{1}, x_{2}, x_{3}, x_{4}\right) R \cap\left(x_{1}, x_{3}, x_{4}, x_{5}\right) R}
$$

shows that $\Gamma_{\left(x_{2}, x_{5}\right) R}(H)=0$ and $H_{\left(x_{2}, x_{5}\right) R}^{1}(H)=E_{R}(R / m)$. On the other hand, for $p:=\left(x_{1}, x_{3}, x_{4}, x_{5}\right) R$, we have

$$
H_{x_{2}}=H_{p R_{x_{2}}}^{4}\left(R_{x_{2}}\right)=H_{p R_{p}}^{4}\left(R_{p}\right)=E_{R}\left(R /\left(x_{1}, x_{3}, x_{4}, x_{5}\right) R\right)
$$

(because $H_{p R_{x_{2}}}^{4}\left(R_{x_{2}}\right)$ has a natural $R_{p}$-module structure). Similarly, $H_{x_{5}}=$ $E_{R}\left(R /\left(x_{1}, x_{2}, x_{3}, x_{4}\right)\right) R$. Together with (+) our claim follows. Thus,

$$
\operatorname{injdim}_{R}(H)=\operatorname{dim}_{R}(H)=1 \text {. }
$$

- $s=2, b=2, d=6$. We take $I:=\left(x_{1}, x_{2}\right) R \cap\left(x_{3}, x_{4}\right) R \cap\left(x_{5}, x_{6}\right) R$ and $H:=H_{I}^{4}(R)$. Again, because of Remark 2.10, $H$ is not $I$-cofinite (it is not zero because, by Lyubeznik's result mentioned above, $\operatorname{cd}(I, R)=6-s=4)$. A Mayer-Vietoris sequence argument with respect to the ideals $\left(x_{1}, x_{2}\right) R \cap$ $\left(x_{3}, x_{4}\right) R$ and $\left(x_{5}, x_{6}\right) R$ shows that there is a canonical isomorphism $H=$ $H_{\left(x_{1}, x_{2}, x_{5}, x_{6}\right) R \cap\left(x_{3}, x_{4}, x_{5}, x_{6}\right) R}^{5}(R)$. From this it is easy to see that

$$
\operatorname{injdim}_{R}(H)=\operatorname{dim}_{R}(H)=0 .
$$

- $s=2, b=2, d=6$. We take $I:=\left(x_{1}, x_{2}\right) R \cap\left(x_{3}, x_{4}\right) R \cap\left(x_{5}, x_{6}\right) R$ and $H:=H_{I}^{3}(R)$. Again, because of Remark 2.10, $H$ is not $I$-cofinite (we will see below that it is not zero). A similar Mayer-Vietoris sequence as in the previous example provides us with a short exact sequence

$$
\begin{gathered}
\quad 0 \rightarrow H_{\left(x_{1}, x_{2}, x_{3}, x_{4}\right) R}^{4}(R) \rightarrow H \\
\rightarrow H_{\left(x_{1}, x_{2}, x_{5}, x_{6}\right) R}^{4}(R) \oplus H_{\left(x_{3}, x_{4}, x_{5}, x_{6}\right) R}^{4}(R) \rightarrow 0 .
\end{gathered}
$$

On the other hand, the spectral sequence belonging to the composed functors $\Gamma_{m} \circ \Gamma_{\left(x_{1}, x_{2}, x_{5}, x_{6}\right) R}$ shows that $H_{m}^{2}\left(H_{\left(x_{1}, x_{2}, x_{5}, x_{6}\right) R}^{4}(R)\right)=H_{m}^{6}(R) \neq 0$. This fact together with the above exact sequence implies $H_{m}^{2}(H) \neq 0$; therefore we have

$$
\operatorname{injdim}_{R}(H)=\operatorname{dim}_{R}(H)=2 .
$$

- $s=2, b=3, d=7$. We take $I:=\left(x_{1}, x_{2}, x_{3}\right) R \cap\left(x_{4}, x_{5}, x_{6}\right) R \cap\left(x_{7}, x_{1}, x_{2}\right) R$ and $H:=H_{I}^{5}(R)$. Because of Remark 2.10, $H$ is not $I$-cofinite. Similar arguments as in the first example show

$$
\operatorname{injdim}_{R}(H)=\operatorname{dim}_{R}(H)=1
$$

(one can start e.g. with the Mayer-Vietoris sequence belonging to the ideals $\left(x_{1}, x_{2}, x_{3}\right) R \cap\left(x_{7}, x_{1}, x_{2}\right) R$ and $\left.\left(x_{4}, x_{5}, x_{6}\right) R\right)$.

- $s=2, b=3, d=7$. We take $I:=\left(x_{1}, x_{2}, x_{3}\right) R \cap\left(x_{4}, x_{5}, x_{6}\right) R \cap\left(x_{7}, x_{1}, x_{4}\right) R$ and $H:=H_{I}^{5}(R)$. Because of Remark 2.10, $H$ is not $I$-cofinite. The MayerVietoris sequence with respect to the ideals $\left(x_{1}, x_{2}, x_{3}\right) R \cap\left(x_{7}, x_{1}, x_{4}\right) R$ and $\left(x_{4}, x_{5}, x_{6}\right) R$ provides us with an exact sequence

$H_{\left(x_{1}, x_{2}, x_{3}\right) R \cap\left(x_{7}, x_{1}, x_{4}\right) R}^{5}(R) \rightarrow H \rightarrow H_{\Sigma}^{6}(R) \rightarrow H_{\left(x_{1}, x_{2}, x_{3}\right) R \cap\left(x_{7}, x_{1}, x_{4}\right) R}^{6}(R)$. 
Here

$$
\begin{aligned}
\Sigma & :=\left(\left(x_{1}, x_{2}, x_{3}\right) R \cap\left(x_{7}, x_{1}, x_{4}\right) R\right)+\left(x_{4}, x_{5}, x_{6}\right) R \\
& =\left(x_{1}, \ldots, x_{6}\right) R \cap\left(x_{1}, x_{4}, x_{5}, x_{6}, x_{7}\right) R .
\end{aligned}
$$

Note that, by an obvious Mayer-Vietoris sequence argument, one has

$$
H_{\left(x_{1}, x_{2}, x_{3}\right) R \cap\left(x_{7}, x_{1}, x_{4}\right) R}^{l}(R)=0
$$

for every $l \geq 5$; therefore, one gets a canonical isomorphism

$$
H=H_{\Sigma}^{6}(R)
$$

The height of $\Sigma$ is five and so $H_{\Sigma}^{l}(R)=0$ for $l \notin\{5,6\}$. In particular, $H_{x_{7} R}^{p}\left(H_{\Sigma}^{q}(R)\right)$ is zero if $q \notin\{5,6\}$ or if $p>1$ (i.e., there are only four potentially nonzero terms) and thus the convergent spectral sequence

$$
E_{2}^{p, q}:=H_{x_{7} R}^{p}\left(H_{\Sigma}^{q}(R)\right) \Rightarrow H_{x_{7} R+\Sigma}^{p+q}(R)=H_{\left(x_{1}, x_{4}, x_{5}, x_{6}, x_{7}\right) R}^{p+q}(R)
$$

shows $\Gamma_{x_{7} R}(H)=0$ (because $\Gamma_{x_{7} R}(H)=E_{2}^{0,6}=E_{\infty}^{0,6}$ and even $E_{\infty}^{6}=$ $H_{\left(x_{1}, x_{4}, x_{5}, x_{6}, x_{7}\right) R}^{6}=0$ ) and $H_{x_{7} R}^{1}(H)=0$ (because $H_{x_{7} R}^{1}(H)=E_{2}^{1,6}=$ $\left.E_{\infty}^{1,6}=E_{\infty}^{7}=H_{\left(x_{1}, x_{4}, x_{5}, x_{6}, x_{7}\right) R}^{7}=0\right)$. The two latter vanishing results mean precisely that the canonical map $H \rightarrow H_{x_{7}}$ is an isomorphism. On the other hand,

$$
(H=) H_{x_{7}}=H_{\Sigma}^{6}(R)_{x_{7}}=H_{\left(x_{1}, \ldots, x_{6}\right) R}^{6}(R)_{x_{7}} .
$$

On the other hand, it is clear that

$$
\operatorname{Supp}_{R}\left(H_{\left(x_{1}, \ldots, x_{6}\right) R}^{6}(R)\right)=\left\{\left(x_{1}, \ldots, x_{6}\right) R,\left(x_{1}, \ldots, x_{7}\right) R\right\} ;
$$

in fact, a more precise statement is possible:

$\operatorname{Ext}_{R}^{p}\left(R /\left(x_{1}, \ldots, x_{6}\right) R, H_{\left(x_{1}, \ldots, x_{6}\right) R}^{6}(R)\right)=\operatorname{Ext}_{R}^{p+6}\left(R /\left(x_{1}, \ldots, x_{6}\right) R, R\right)$

and

$\operatorname{Ext}_{R}^{p}\left(R /\left(x_{1}, \ldots, x_{7}\right) R, H_{\left(x_{1}, \ldots, x_{6}\right) R}^{6}(R)\right)=\operatorname{Ext}_{R}^{p+6}\left(R /\left(x_{1}, \ldots, x_{7}\right) R, R\right)$

$(p \in \mathbb{N}$ is arbitrary) are isomorphisms that come from obvious spectral sequence arguments; the Bass numbers of $R$ itself are known (as $R$ is Gorenstein) and so one may conclude that a minimal injective resolution of $H_{\left(x_{1}, \ldots, x_{6}\right) R}^{6}(R)$ has the form

$0 \rightarrow H_{\left(x_{1}, \ldots, x_{6}\right) R}^{6}(R) \rightarrow E_{R}\left(R /\left(x_{1}, \ldots, x_{6}\right) R\right) \rightarrow E_{R}\left(R /\left(x_{1}, \ldots, x_{7}\right) R\right) \rightarrow 0$.

One may localize this exact sequence in $x_{7}$ and derive canonical isomorphisms

$$
(H=) H_{\left(x_{1}, \ldots, x_{6}\right) R}^{6}(R)_{x_{7}}=E_{R}\left(R /\left(x_{1}, \ldots, x_{6}\right) R\right) .
$$

Now it is clear that one has

$$
\operatorname{injdim}_{R}(H)=0 \neq 1=\operatorname{dim}_{R}(H) .
$$

Summary 2.12. Let $R$ be a Noetherian local regular ring containing a field, $I$ an ideal of $R$ and $l$ a natural number. Here is a summary of the previous results: 2.6).

(i) If $H_{I}^{l}(R)$ is $I$-cofinite, then $\operatorname{inj}_{\operatorname{dim}_{R}}\left(H_{I}^{l}(R)\right)=\operatorname{dim}_{R}\left(H_{I}^{l}(R)\right)$ holds (Corollary

(ii) There are examples where $H_{I}^{l}(R)$ is not $I$-cofinite, but $\operatorname{injdim}_{R}\left(H_{I}^{l}(R)\right)=$ $\operatorname{dim}_{R}\left(H_{I}^{l}(R)\right)$ holds (e.g., the first example from 2.11). 
(iii) There are examples where $\operatorname{inj}_{\operatorname{dim}}\left(H_{I}^{l}(R)\right)=\operatorname{dim}_{R}\left(H_{I}^{l}(R)\right)$ does not hold (e.g., the last example from 2.11, where $l=\operatorname{cd}(I, R)$ ).

Question 2.13. Does there exist a Cohen-Macaulay ideal $I$ in a regular local ring $R$ containing a field of characteristic zero for which $\operatorname{injdim}_{R}\left(H_{I}^{l}(R)\right)=\operatorname{dim}_{R}\left(H_{I}^{l}(R)\right)$ does not hold for some natural $l$ ?

\section{REFERENCES}

[1] Delfino, D. and Marley, T., Cofinite modules and local cohomology, J. Pure and Appl. Alg. 121, (1997) 45-52. MR1471123 (98g:13015)

[2] Faltings, G., Über lokale Kohomologiegruppen hoher Ordnung, J. für die reine und angewandte Mathematik 313, (1980) 31-51. MR552461 (82f:14006)

[3] Grothendieck, A., Local Cohomology, Lecture Notes in Mathematics, Springer-Verlag, (1967). MR0224620 (37:219)

[4] Hartshorne, R., Affine duality and cofiniteness, Inv. Math. 9, (1969/1970) 145-164. MR0257096 (41:1750)

[5] Huneke, C. and Koh, J., Cofiniteness and vanishing of local cohomology modules, Math. Proc. Camb. Phil. Soc. 110, (1991) 421-429. MR1120477 (92g:13021)

[6] Huneke, C. and Lyubeznik, G., On the vanishing of local cohomology modules, Invent. Math. 102, (1990) 73-93. MR 1069240 (91i:13020)

[7] Kawasaki, K.-I., Cofiniteness of local cohomology modules for principal ideals, Bull. London Math. Soc. 30, (1998) 241-246. MR.1608094 (98m:13025)

[8] Lyubeznik, G., Some algebraic sets of high local cohomological dimension in projective space, Proc. Amer. Math. Soc. 95, (1985) 9-10. MR796437 (87c:14005)

[9] Lyubeznik, G., Finiteness properties of local cohomology modules (an application of $D$ modules to commutative algebra), Invent. Math. 113, (1993) 41-55. MR1223223|(94e:13032)

[10] Lyubeznik, G., $F$-modules: Applications to local cohomology and $D$-modules in characteristic $p>0$, J. Reine Angew. Math. 491, (1997) 65-130. MR:1476089 (99c:13005)

[11] Marley, T. and Vassilev, J. C., Cofiniteness and associated primes of local cohomology modules, Journal of Algebra 256, (2002) 180-193. MR1936885 (2003j:13025)

[12] Yoshida, K.-I., Cofiniteness of local cohomology modules for ideals of dimension one, Nagoya Math. J. 147, (1997) 179-191. MR1475172(98j:13014)

Fakultät für Mathematik und Informatik, Universität Leipzig, PF 1009 20, D-04009 LeIPZig, Germany

E-mail address: hellus@math.uni-leipzig.de 